\title{
Design of 'Smart' pebble for sediment entrainment study
}

\author{
A. Dwivedi ${ }^{1}$, B. Melville ${ }^{1}$, E. Akeila ${ }^{2}$ \& Z. Salcic ${ }^{2}$ \\ ${ }^{1}$ Department of Civil \& Environmental Engineering, \\ The University of Auckland, Auckland, New Zealand \\ ${ }^{2}$ Department of Electrical and Computer Engineering, \\ The University of Auckland, Auckland, New Zealand
}

\begin{abstract}
The 'Smart' pebble is a small self-equipped datalogger having a $40 \mathrm{~mm}$ outer diameter with a built-in sensor for acceleration and rotation measurements. This pebble has the capability to detect instantaneous acceleration due to turbulence in fluid, which is indicative of hydrodynamic forces acting on the pebble. The pebble stores the data continuously in its internal memory. These data can subsequently be transferred by serial port to a computer for detailed analysis. Test runs using the smart pebble have been carried out to validate accurate functioning of the acceleration and gyro sensor. The acceleration of the smart pebble was compared to the acceleration of the shake table. The results show the excellent capability of the pebble to record the instantaneous forces acting on the pebble during its entrainment.
\end{abstract}

Keywords: entrainment, hydrodynamic forces, accelerometer, gyroscope.

\section{Introduction}

In order to understand the processes causing particle entrainment, the stability of the separate particles must be evaluated. The hydrodynamic forces on the particles are very important for understanding this stability. Figure 1 (a) shows the hydrodynamic forces acting on the sediment particle. Figure 1 (b) shows the test arrangement of smart pebble.

This research has been aimed at improving the present level of understanding of the physical processes involved in the transport of individual sediment particles in the near bed region of a turbulent open channel flow by the use of smart pebble. 


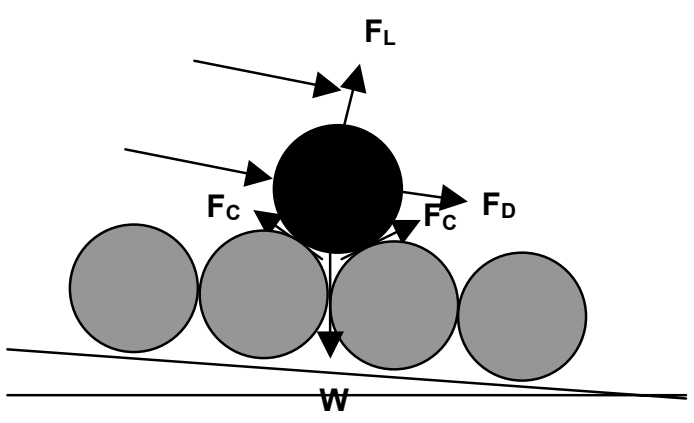

(a)

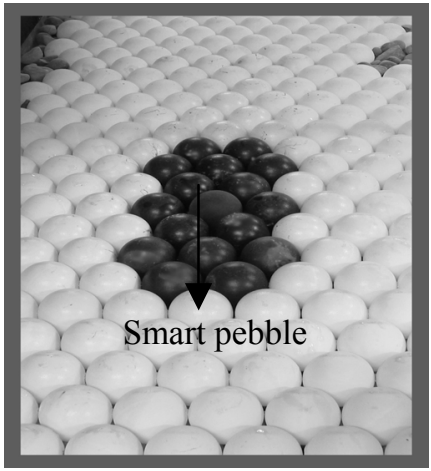

(b)

Figure 1: $\quad$ (a) Hydrodynamic forces on the particle where $F_{L}$ is lift force, $F_{D}$ is drag force, $\mathrm{F}_{\mathrm{C}}$ contact force and $\mathrm{W}$ the weight of pebble. (b) Test arrangement using the smart pebble inside the flume.

\section{Background}

Inertial navigation system (INS) concepts (Titerron and Weston [3]) are used as the basis of this work. An INS is a system, which can determine its exact location in three-dimensional space, relative to a known starting location. The location is resolved by integrating the accelerations and rotations about the three axes. Three accelerometers and three gyroscopes measure the accelerations and angular rotations about the three axes, giving the particle six degrees of freedom. The reference frame consisting of three axes of the body of the pebble is known as the Body Frame. The set of sensor modules, organized in orthogonal form, measure the accelerations and gyrations with reference to the body frame. As the forces acting on the pebble need to be measured relative to a stationary frame of reference a conversion is necessary.

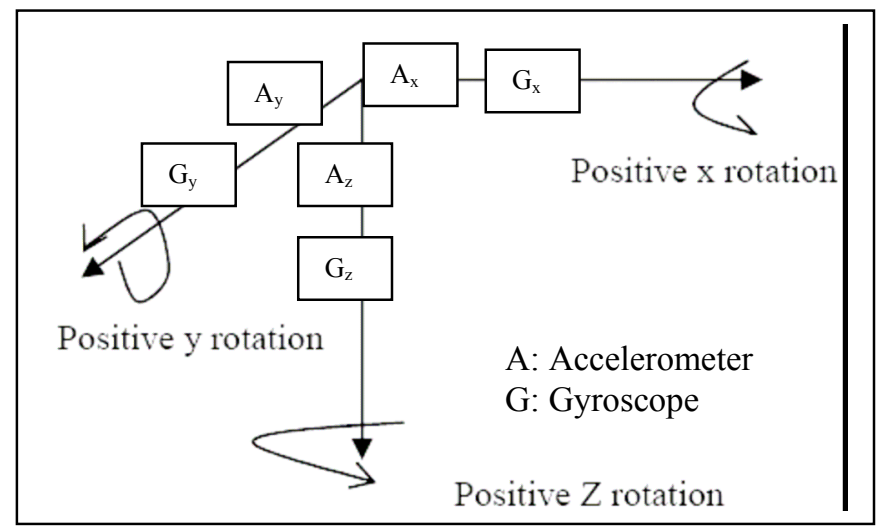

Figure 2: $\quad$ Axis conversion from body frame to reference frame. 
There are several methods of performing a coordinate transformation (Titerron and Weston [3]). The Euler Angle method (Pio [4]) was chosen as the most appropriate method, given the processing and time constraints in this proof of concept project.

The Euler Angle method performs the coordinate transformation as three rotations about the three axes. To transform the body frame related motion parameters to the reference frame, three angular rotations, $\psi, \theta$ and $\varphi$ are performed around the $\mathrm{z}, \mathrm{y}$ and $\mathrm{x}$ axes respectively. When the three rotations are performed as per Figure 2, we get three matrices $C 1, C 2, C 3$, representing the rotations as below:

Rotation $\psi$ about the reference $\mathrm{Z}$ axis,

$$
C_{1}=\left[\begin{array}{ccc}
\operatorname{Cos}(\psi) & \operatorname{Sin}(\psi) & 0 \\
-\operatorname{Sin}(\psi) & \operatorname{Cos}(\psi) & 0 \\
0 & 0 & 1
\end{array}\right]
$$

Rotation $\theta$ about the new y-axis,

$$
C_{2}=\left[\begin{array}{ccc}
\operatorname{Cos}(\theta) & 0 & -\operatorname{Sin}(\theta) \\
0 & 1 & 0 \\
\operatorname{Sin}(\theta) & 0 & \operatorname{Cos}(\theta)
\end{array}\right]
$$

Rotation $\varphi$ about the new $\mathrm{x}$-axis,

$$
C_{3}=\left|\begin{array}{ccc}
1 & 0 & 0 \\
0 & \operatorname{Cos}(\varphi) & \operatorname{Sin}(\varphi) \\
0 & -\operatorname{Sin}(\varphi) & \operatorname{Cos}(\varphi)
\end{array}\right|
$$

The three individual matrices can be combined into one complete matrix as follows

$$
C_{n}^{b}=C_{3} \bullet C_{2} \bullet C_{1}
$$

The complete matrix $C_{n}^{b}$ is known as the rotation matrix

The conversion of any vector from the body frame to the reference frame is achieved by the multiplication of the body frame vector by the transpose of the rotation matrix. This is indicated in the following:

$$
r^{n}=C_{b}^{n} \bullet r^{b}
$$

$r^{n}$ is the vector in reference frame,

$C_{b}^{n}$ is the transpose of the rotation matrix $C_{n}^{b}$, and

$r^{b}$ is the vector in the body frame 
For the order of rotation in Equation (5) the transpose of the rotation matrix $C_{n}^{b}$ is as follows.

$C_{b}^{n}=\left[\begin{array}{ccc}\operatorname{Cos}(\theta) \operatorname{Cos}(\psi) & -\operatorname{Cos}(\varphi) \operatorname{Sin}(\psi)+\operatorname{Sin}(\varphi) \operatorname{Sin}(\theta) \operatorname{Cos}(\psi) & \operatorname{Sin}(\varphi) \operatorname{Sin}(\psi)+\operatorname{Cos}(\varphi) \operatorname{Sin}(\theta) \operatorname{Cos}(\psi) \\ \operatorname{Cos}(\theta) \operatorname{Sin}(\psi) & \operatorname{Cos}(\varphi) \operatorname{Cos}(\psi)+\operatorname{Sin}(\varphi) \operatorname{Sin}(\theta) \operatorname{Sin}(\psi) & -\operatorname{Sin}(\varphi) \operatorname{Cos}(\psi)+\operatorname{Cos}(\varphi) \operatorname{Sin}(\theta) \operatorname{Sin}(\psi) \\ -\operatorname{Sin}(\theta) & \operatorname{Sin}(\varphi) \operatorname{Cos}(\theta) & \operatorname{Cos}(\varphi) \operatorname{Cos}(\theta)\end{array}\right]$

Between successive updates in the real time computation of body attitude there will be small change in angle rotations such that

$\sin \phi \rightarrow \phi, \sin \theta \rightarrow \theta, \sin \psi \rightarrow \psi$ and the cosine of these angles approach unity. Making these substitutions in equation (6) and ignoring the product of angles, which also become small, the direction matrix expressed in terms of Euler rotations reduces approximately to the skew symmetric form

$$
C_{b}^{n}=\left[\begin{array}{ccc}
1 & -\psi & \theta \\
\psi & 1 & -\phi \\
-\theta & \phi & 1
\end{array}\right]
$$

In such a situation, if commercial off the shelf (COTS) accelerometers and gyroscope ICs are used to monitor instantaneous motion parameters, $r^{b}$ vector can be indicated as,

$$
r b=\left[\begin{array}{c}
A_{x-b} \\
A_{y-b} \\
A_{z-b}
\end{array}\right]
$$

where $\mathrm{Ax}_{-b}, A_{y-b}$ and $A_{z-b}$ are the body frame accelerations in the corresponding axes.

This gives us the simplified relationship for the $r^{n}$ vector in the reference frame as,

$$
r^{n}=\left[\begin{array}{ccc}
1 & -\psi & \theta \\
\psi & 1 & -\phi \\
-\theta & \phi & 1
\end{array}\right]\left[\begin{array}{c}
A_{x-b} \\
A_{y-b} \\
A_{z-b}
\end{array}\right]
$$

For the angular rotation parameters, the following relationships were used.

$$
\psi=\int \omega_{z} d t, \quad \theta=\int \omega_{y} d t, \phi=\int \omega_{x} d t
$$

where $\omega_{x}, \omega y$ and $\omega_{z}$ are the gyrations in respective axes.

\section{Design considerations}

Key design considerations for smart pebble are packaging, powering, relative physical placement of the MEMS, signal conditioning and providing a bare minimum digital subsystem and a compact and simple data communication 
interface. Packaging has direct influence on the size of the pebble. Since roughness elements involved in the sediment entrainment study are $40 \mathrm{~mm}$ diameter ping pong balls, the diameter of the pebble should not be more than $40 \mathrm{~mm}$, which is only possible with proper compact packaging of the electronics involved. Entrainment is a sporadic and instantaneous event. The time at which particle will be entrained can vary from few seconds to few minutes. This directly put constraint on powering the smart pebble for at least 15 minutes to capture the meaningful data before entrainment, during entrainment and after entrainment of the pebble. Physical placement of the sensors and electronics should be such that the centre of gravity is at the centre of the sphere (pebble) to avoid any abrupt motion due to eccentricity of the inside mass. Compact and simple data communication system was required to make the pebble userfriendly.

\section{Sensors}

In order to monitor the accelerations, commercial off the shelf (COTS) type accelerometers and gyroscope MEMS from Analog Devices were considered based on their sensitivity, accuracy, noise behaviour and the offset, etc (Brown et al [1]). ADXL202 dual axis accelerometers and ADXRS150 yaw rate gyroscopes were found suitable. These devices were placed in three orthogonal sensor modules with suitable signal conditioning circuitry.

The unit is powered by a $6 \mathrm{~V}$ battery, which generally powers the circuit up to about 15 minutes continuously. A Texas Instruments TPS60132 charge pump has been utilized to regulate the supply voltage of the pebble at $5 \mathrm{~V}$. Additionally, it boosts up the battery voltage as it dips below $5 \mathrm{~V}$ to maintain the accuracy of the sensor readings. The entire system works on $5 \mathrm{~V}$, except for the external flash memory, which requires $3 \mathrm{~V}$ supplied through a REG102 $3 \mathrm{~V}$ regulator.

Since dual gang accelerometers were used, it was possible to take all the necessary measurements with two chips to keep the real estate of the PCBs to a minimum. Each sensor produces an analogue voltage proportional to the acceleration or gyration measured. These outputs are periodically scanned and averaged out to minimize noise, through the analogue-to-digital-converter channels of the Microchip PIC18F8520 microcontroller. The digitized sensor data is then stored in an external flash memory, SST25VF020. Since the data is collected at regular time intervals, this data is later processed to determine the accelerations of the pebble.

The smart pebble is a complete, battery powered unit equipped with two dualaxis accelerometers and three angular rate sensors, a microcontroller, nonvolatile external memory and a user interface capable of communication with a PC. It has been packaged into a sphere of $40 \mathrm{~mm}$ diameter as illustrated in Figure 3.

The stored data is transferred to a PC over the hyper terminal of the computer. This data is then processed in MATLAB to calculate the true accelerations along the reference axes. The MATLAB program takes in the raw digitized sensor data and converts them to the analogue voltage values measured originally. Since the fixed axis set of the sensor boards are subject to rotations, the accelerations 


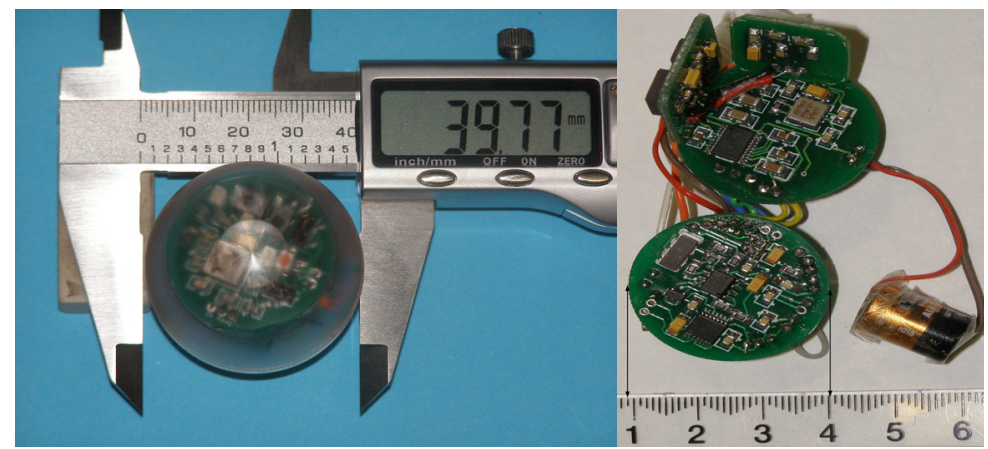

Figure 3: Final packaged 'smart pebble'. Right: Actual size of the PCBs. Left: The PCBs inside the shell of the smart pebble.

measured are in fact, not the true accelerations along a set of reference axes. Therefore the data needs some complex mathematical processing to extract the true acceleration data. The gyroscope measurements are integrated to obtain the rotation angles of the unit, about each axis. Next, the initial tilt of the unit and the gravity components along each axis are calculated. Then the direction cosine matrix and Euler angles are used to convert the data to obtain the true accelerations along each reference axis. Finally gravity is compensated for and the accelerations along the reference axes are plotted.

The sensors are mounted such that the accelerations along the three orthogonal axes and the gyrations about each axis are measured.

\section{Operation of the smart pebble}

There are two switches on the sensors, power switch and a Data protection switch (protection for the stored sensor data). Data protection switch is coupled with a push button, which triggers the interrupt to start reading the sensor data and storing them in the flash. Turn this switch on and push the button next to it. This should briefly light a blue LED. This means the processor will start reading the data off the sensors in one minute. Once the interrupt has been triggered (i.e. blue LED) turn the switch off.

This means that there will be no accidental triggering of the interrupts once we have set it out. The main reason for doing this is, each time we need to store data in the flash and retrieve data out to pc and then erase the chip for the next set of Data. So if the interrupt is triggered on the way all the data stored up until then will be lost. There is a second push button (also coupled to an LED) to retrieve data off the micro, into the $\mathrm{PC}$

\section{Calibration and testing}

In developing a system of this nature, the designer has to be aware of the overall errors contributed by many sources. Some obvious errors are the accelerometer 
offsets, OpAmp offsets, the analog calculation interface inaccuracies and offsets and noise from the sensors.

Calibration was performed in two steps. In the first step individual sensors were calibrated and in the second step complete smart pebble system was calibrated.

\subsection{Individual sensor calibration}

The accelerometer sensors were calibrated against the gravity by orienting the axes of the sensors towards the gravitational direction to get $+\mathrm{g}$ output and then opposite to that direction to get $-\mathrm{g}$. After that, each sensor gain and offset is calculated using the following equations.

$$
\begin{aligned}
& \operatorname{offset}(V)=\frac{V_{+g}+V_{-g}}{2} \\
& \operatorname{Gain}(V / g)=V_{+g}-\text { offset }
\end{aligned}
$$

The gyroscope calibration was carried out by placing each gyroscope on a servo motor. The motor moves in a controlled sequence $\left( \pm 180^{\circ}\right)$. The rotational speed of the servo motor has been adjusted so that it does not exceed the gyroscope maximum sensitivity $\left(150^{\circ} / \mathrm{s}\right)$. This was achieved by controlling the voltage level that supplies the motor (at $3 \mathrm{~V}$ the speed is $83^{\circ} / \mathrm{s}$ ). The outputs of the gyroscopes are observed using an oscilloscope. When the motor rotates in the positive direction relative to the gyroscope sensitive axis, the output of the sensor gives a high pulse voltage with respect to the sensor's offset, and the opposite happens when the motor rotates in the reverse direction. The sensitivity (gain) of the gyroscope is calculated using equation 10.

$$
\operatorname{Gain}\left(V /{ }^{o} / \mathrm{s}\right)=\frac{\left(V_{+}-V_{\text {offset }}\right) \Delta \mathrm{t}}{180}
$$

\subsection{Overall system calibration}

Overall system calibration was done with shake table. For this set of calibration a hollow cube was made in which the sensors can be in housed. The advantage of using cube is that it can be placed on the shake table with the direction of the shaking coinciding with the direction of the acceleration sensors. The shake table is fitted with an accelerometer that records the acceleration of the table. After giving the sine wave motion to the shake table for 30 seconds, the accelerations were compared to the accelerations recorded by the smart pebble. This was done for all the three axis of the pebble coinciding with the motion of the shake table. Similar experiments were performed with the cube placed at 45 degrees to the shake table motion

The first step in the calibration process was testing the zero acceleration output (Null Offset). The total noise included in this version of the smart pebble has caused an accumulation error when implementing digital mathematical integration on the outputs of the gyroscopes. Figure 4 shows the output from the 
smart pebble when it was left stationary for about 15 minutes while Figure 5 shows the accumulative angle calculation error.

The null offset output has been improved by filtering the data using some MATLAB built-in digital filters and taking the average of the first 5 seconds of each sensor output as reference value for calculating the accelerations and angles. Figure 5 shows the output data resulted from filtering and averaging.

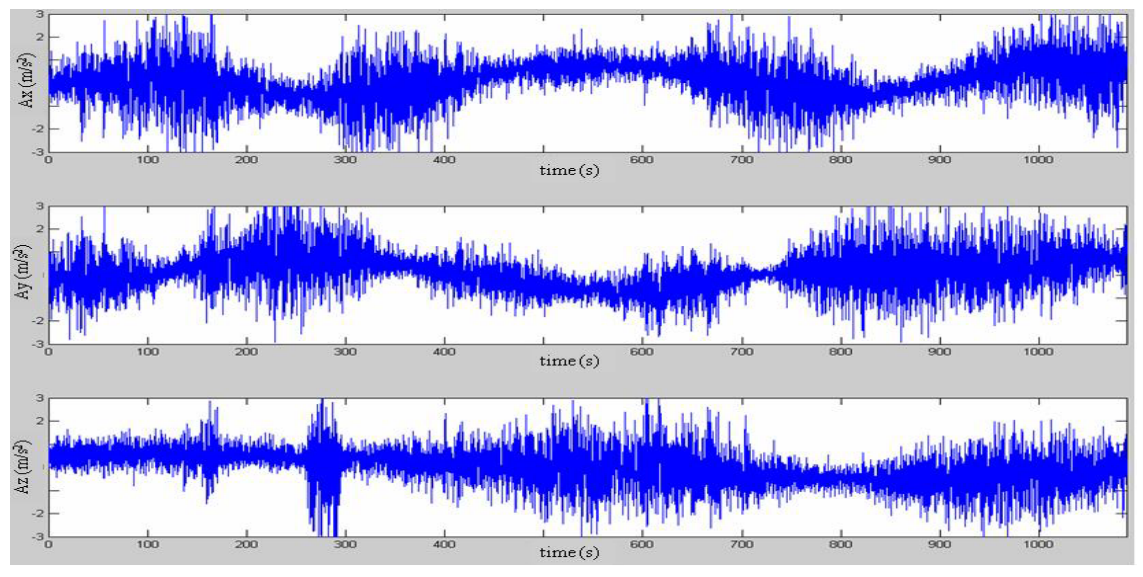

Figure 4: Acceleration resulted from the smart pebble when it is left stationary.

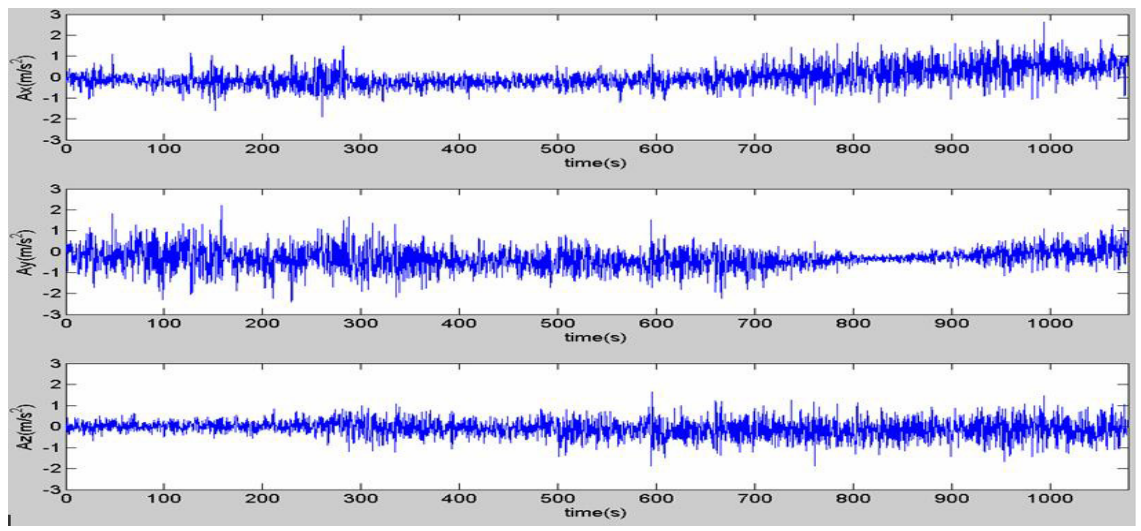

Figure 5: Smart pebble stationary output after filtering and averaging (acceleration).

Because of the low sampling rate $(8.33 \mathrm{~Hz})$, frequency of motions was selected to be less than $4 \mathrm{~Hz}$ to avoid signal aliasing. The preliminary results show that the pebble can accurately detect the frequency of the applied motions. 
However, due to the noise and mainly the very low sampling frequency, the amplitude output error is very high (it ranges from $63 \%$ to more than $92 \%$ in some time intervals. To reduce the amplitude error we tested the smart pebble at different sampling frequency and found that the error can be reduced to almost $5 \%$ if sampling is done at more that ten times the required motion's frequency.

Figure 6 and 7 shows the affect of sampling frequency on smart pebbles output. It can be seen that the amplitude error is minimised by increasing the sampling frequency (Figure 7).

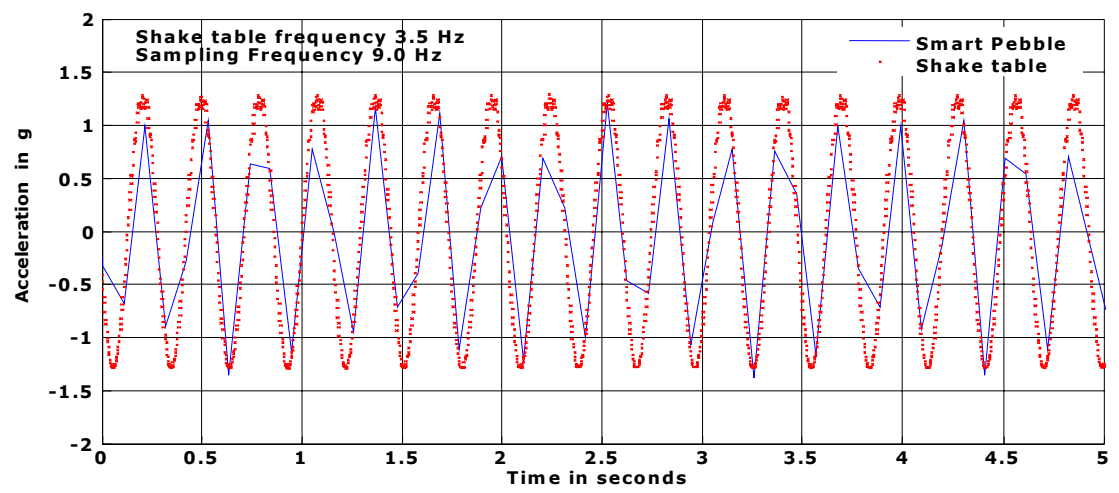

Figure 6: $\quad$ Smart pebble output versus the shake table (sampling frequency $9 \mathrm{~Hz}$ )

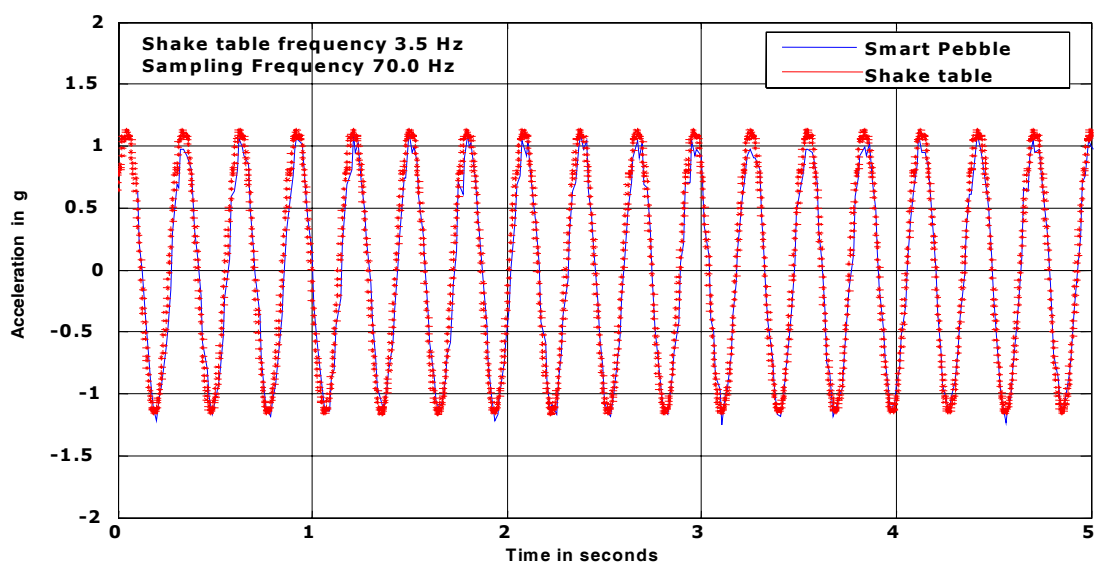

Figure 7: Smart pebble output versus the shake table (sampling frequency $70 \mathrm{~Hz})$.

\section{Conclusions}

Initial testing and calibration of smart pebble shows the excellent functioning and its capabilities to log instantaneous accelerations. Few measurements of the smart pebble were done inside the flume with water and data analysis is in 
progress. Further work in reducing the overall power consumption by selecting a lower power microcontroller is in progress, because the microcontroller in this version of the smart pebble is consuming more than $80 \%$ of the whole power. Work in reducing the noise to signal ratio is in progress. Work is in progress to reduce the size of the pebble by using three axis accelerometers e.g. (Tieman et al [2])

\section{References}

[1] Brown, T.G et al; " Strap-down micromechanical (MEMS) sensors for highG ammunition applications"; IEEE Trans on Magnetics, Vol. 37, No 1, Jan 2001

[2] Tieman, J. et al; "Design of a MEMS based 3-axis accelerometer smart sensor"; Proc of IEEE Sensors for Industry Conf, USA, Nov 2002.

[3] Titerron, D.H. \& Weston, J.L.: " Strapdown inertial navigation technology"; IEEE, 1997

[4] Pio, R: "Euler angle transformations"; IEEE Transactions on Automatic Control, Volume 11, Issue 4, Oct 1966. 